\title{
Objective photographic assessments and comparisons of immediate bilateral breast reconstruction using deep inferior epigastric perforator flaps and implants
}

\author{
Hyun Ho Han, Jin Mi Choi, Jin Sup Eom \\ Department of Plastic Surgery, Asan Medical Center, University of Ulsan College of Medicine, Seoul, Korea
}

Background The increasing number of bilateral breast cancer patients has been accompanied by a growing need for bilateral mastectomy with immediate reconstruction. However, little research has investigated the complications and aesthetic outcomes related to bilateral reconstruction. Therefore, we analyzed retrospective data comparing the outcomes of bilateral reconstruction using deep inferior epigastric perforator (DIEP) flaps or implants.

Methods This study included 52 patients (24 DIEP group and 28 implant group) who underwent bilateral mastectomy with immediate reconstruction between 2010 and 2020. Patient demographics, surgical characteristics, and complications were recorded. The difference between the left and right position of the nipple-areolar complex with respect to the sternal notch point at the clavicle was measured, and breast symmetry was evaluated.

Results The average weight of breasts reconstructed with DIEP flaps $(417.43 \pm 152.50 \mathrm{~g})$ was higher than that of breasts with implants. The hospitalization period and operation time were significantly longer in the DIEP group. Early complications were significantly more common in the implant group (36.53\%) than in the DIEP group. The angles between the nipples and the horizontal line were $1.09^{\circ} \pm 0.71^{\circ}$ and $1.75^{\circ} \pm 1.45^{\circ}$ in the DIEP and implant groups, respectively. Conclusions Although the surgical burden is lower, breast reconstruction using implants requires greater attention with respect to implant positioning, asymmetry, and complications than DIEP flap reconstruction. DIEP flap reconstruction has a prolonged operation time and a high risk of flap failure, but yields excellent cosmetic results and does not require intensive follow-up. Patients should be consulted to determine the most suitable option for them.

Keywords Breast reconstruction / Breast neoplasms / Perforator flaps / Free tissue flap / Breast implants

\author{
Correspondence: Jin Sup Eom \\ Department of Plastic Surgery, Asan \\ Medical Center, University of Ulsan \\ College of Medicine, 88 Olympic-ro \\ 43-gil, Songpa-gu, Seoul 05505, \\ Korea \\ Tel: +82-2-3010-3600 \\ Fax: +82-2-476-7471 \\ E-mail: jinsupp@amc.seoul.kr
}

Received: November 28, 2020 • Revised: June 19, 2021 • Accepted: July 7, 2021

pISSN: 2234-6163 • elSSN: 2234-6171 • https://doi.org/10.5999/aps.2020.02362 • Arch Plast Surg 2021;48:473-482

\section{INTRODUCTION}

Breast cancer is one of the leading causes of cancer-related death among women, and its incidence is on the rise [1-3]. The number of patients with bilateral breast cancer has also been increas- ing [4-6]. Increased screening for breast cancer and the development of new diagnostic imaging methods have played major roles in this trend $[7,8]$. Consequently, the rate of patients who undergo bilateral mastectomy with immediate reconstruction has also risen $[4,9-11]$. 
Breast cancer-related mutations (especially $B R C A$ ) are another major reason for which patients decide to undergo bilateral mastectomy [12]. The total breast cancer incidence among carriers of the BRCA1 or BRCA2 mutations reaches $87 \%$ by the age of 70 years old [13]. Prophylactic mastectomy of the contralateral breast has therefore become a common treatment approach among unilateral breast cancer patients with $B R C A \mathrm{mu}-$ tations $[9,14]$.

According to statistical data from the Korean Health Insurance Review and Assessment Service, the number of patients treated for breast cancer was 155,135 in 2014, 156,533 in 2015, and 170,192 in 2016, showing an increasing trend every year. Additionally, according to the data, the number of BRCA mutation tests increased by > 10 times from 578 in 2010 to 5,880 in 2017. The increase in the number of BRCA mutation tests in 2016 was linked to the "Angelina Jolie effect" and the activities of various institutions, such as the Korean Hereditary Breast Cancer Research, to promote breast cancer prevention and change popular attitudes. Moreover, public awareness of prophylactic surgery has grown substantially since 2010, and the number of $B R C A$ mutation carriers who undergo prophylactic contralateral mastectomy has increased in turn. Since 2013, the frequency of preventive surgery in South Korea has also increased.

The use of implants when performing bilateral reconstruction has many advantages. It is a simple technique with a short operation time. However, implant reconstruction can lead to potentially unfavorable outcomes for some patients, particularly for women who are obese, have large breasts, have thin breast skin, have connective tissue disorders, are smokers, have undergone prior breast radiotherapy, or are candidates for post-mastectomy radiotherapy [15].

As of this study, research on bilateral breast reconstruction has been limited to examining technical issues related to bilateral deep inferior epigastric perforator (DIEP) flap surgery, outcomes of bilateral DIEP flaps, or outcomes of bilateral implants. No studies comparing the use of implants to autologous tissue in bilateral reconstruction have been conducted.

Bilateral autologous reconstruction is a time-consuming procedure, and the abdomens of slim patients might be insufficient in volume for the procedure to be performed $[16,17]$. However, it is possible to achieve a natural breast shape using this procedure [17]. Autologous breast reconstruction could be used to fill the infraclavicular (upper pole) hollow and anterior axillary fold via a flap in ptotic breasts, which is not possible with implant reconstruction. Additionally, for patients with thin breast skin or large breasts, the incidence of complications after autologous reconstruction is lower than it is for implant reconstruction, especially among patients whose chest walls have been previously irradiated [18].

Only one study has examined patients' satisfaction levels in terms of subjective outcomes after bilateral reconstruction, and no studies have objectively assessed complications and aesthetic outcomes. Therefore, in this study, we retrospectively compared data on implant and autologous reconstruction following bilateral mastectomy. We aimed to test the hypothesis that the demographic characteristics of patients, complications, and cosmetic outcomes differ between the two reconstruction methods.

\section{METHODS}

\section{Demographics}

This retrospective study included 52 women with 104 breasts. All breast cancer patients who underwent bilateral mastectomy with immediate reconstruction at our center between 2010 and 2020 were included. Among them, 26 patients underwent bilateral implant reconstruction and 24 patients underwent bilateral DIEP flap reconstruction. All implants were placed in the subpectoral plane with human acellular dermal matrix $(\mathrm{ADM}) \mathrm{cov}$ erage of the lower pole. Patients with a previous history of surgery for breast cancer, such as breast-conserving surgery, were excluded. Patients who had undergone hybrid reconstruction using autologous tissue in addition to implants were also excluded.

The recorded characteristics of patients were age, height, weight, body mass index (BMI), hypertension, diabetes mellitus, history of smoking or current smoking status, menopause status, chemotherapy, pre-mastectomy or post-mastectomy breast radiation, and the presence of hormone receptors. BMI was calculated as weight $(\mathrm{kg})$ divided by height squared $\left(\mathrm{m}^{2}\right)$. Any patient who had smoked within 6 weeks before surgery was considered to have a previous smoking history.

The weight of the mastectomy specimen and the abdominal flap was analyzed instead of their volume. The final flap weight was measured after the flap was harvested and fully trimmed. Although an analysis using measurements with the same units may have been more valuable, it was prohibitvely difficult to measure flap volume. However, breast tissue and DIEP flaps have almost the same density $\left(0.9954 \mathrm{~g} / \mathrm{cm}^{3}\right.$ and $0.894 \mathrm{~g} / \mathrm{cm}^{3}$, respectively) [11]. Thus, a breast density of $0.9954 \mathrm{~g} / \mathrm{cm}^{3}$ indicates that a mastectomy specimen that weighs $200 \mathrm{~g}$ has a volume of approximately $200.9 \mathrm{~cm}^{3}$. Thus, it was practical to compare the weights of mastectomy specimens, abdominal flaps, and implants using volume. Therefore, in this study, we used cubic centimeters (cc) instead of weight to measure implants. 


\section{Surgical procedure}

The recorded characteristics of surgical procedures included the type of mastectomy (nipple-sparing or skin-sparing), type of axillary surgery (sentinel node biopsy or axillary lymph node dissection), reconstruction method (implant or DIEP), implant size, and weights of the mastectomy specimen and final flap. Details of the operation time and hospitalization period were also collected.

Implant reconstructions were performed as follows. Photographs of all patients were taken and preoperative markings were made to determine breast width and projection. Bilateral implants were chosen and inserted into both breasts after considering both the preoperative markings and the mastectomy specimen weight. The implants used for reconstruction were from Allergan (Allergan, Inc., Irvine, CA, USA) and Mentor (Mentor, Santa Barbara, CA, USA). All surgical plane implants were placed in the subpectoral plane. An ADM was used to resurface and reshape the implant pocket. The ADMs used were CryoDerm (CGBio Co., Seongnam, Korea), Dermacell (LifeNet Health, Virginia Beach, VA, USA), or Megaderm (L\&C BIO, Seongnam, Korea). Two drains were placed inside each pocket.

DIEP flap reconstruction was performed as follows. All patients first underwent preoperative computed tomographic angiography, and preoperative markings were made based on perforator findings. We chose the best and largest perforator around the umbilicus and tried to choose a lateral row perforator rather than a medial row perforator to ensure that it was not located eccentrically. Mastectomy was performed by a breast surgeon before reconstruction surgery. Then, a plastic surgery team took over, and a two-team approach was used during reconstruction surgery. One team prepared the internal mammary vessels to be recipient vessels at the level of the second to the third rib. The other team elevated the DIEP flap. After dividing the pedicle, the flap was weighed and brought to the chest for anastomosis to the internal mammary vessels. The superficial inferior epigastric vein was preserved for all the cases since it may have been needed afterwards as a lifeboat vessel. The right abdominal flap was inset to the left breast, and the left abdominal flap was inset across the right breast. The left abdominal flap was turned counterclockwise so that the tip of the flap pointed to the upper lateral pole of the right chest. Additionally, a part of the umbilicus was situated toward the lateral pole, and the flap was placed slightly obliquely. Similarly, the right flap was placed in the left chest in the same way as the left flap and turned clockwise. The insetting method is depicted in Fig. 1. Next, indocyanine green (ICG) angiography was performed to check perfusion in the flap. In unilateral reconstruction, we focused on contralateral

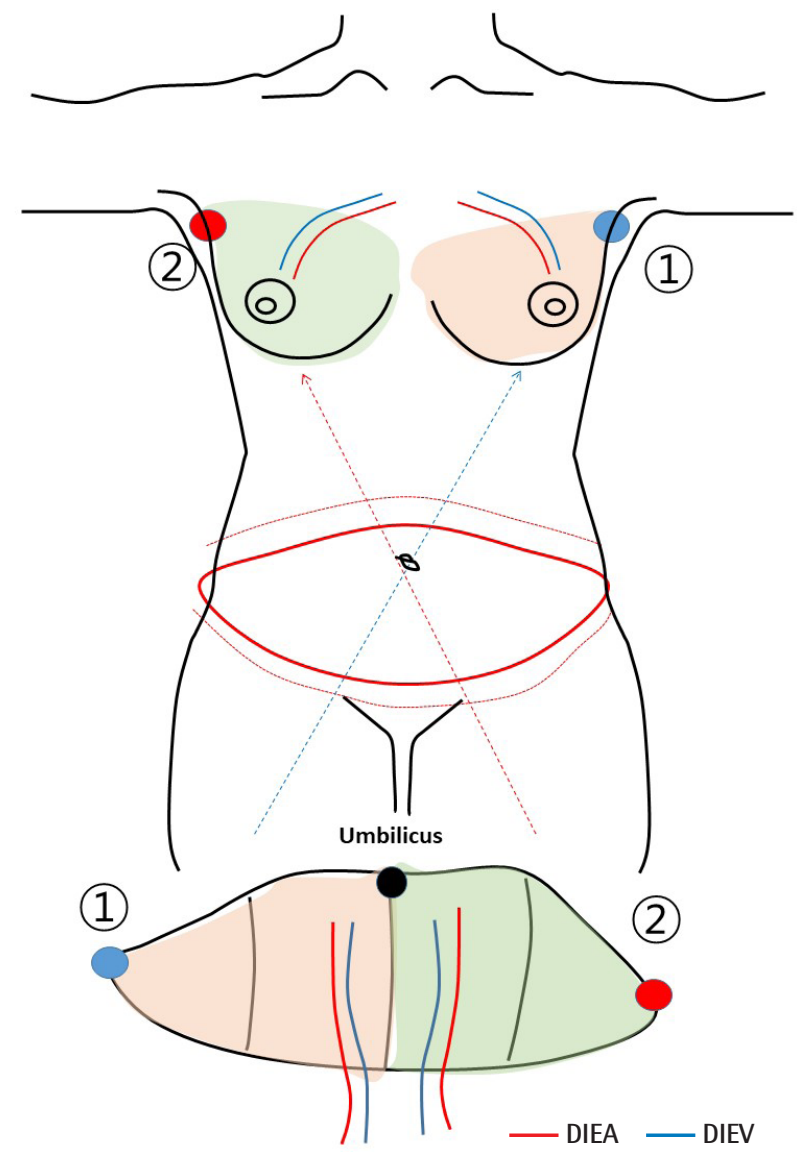

Fig. 1. Depiction of the method for inserting the bilateral deep inferior epigastric perforator flap during surgery. DIEA, deep inferior epigastric artery; DIEV, deep inferior epigastric vein.

perfusion and selected a perforator from the inner row as often as possible, and in bilateral reconstruction, we selected a perforator from the side row. However, using preoperative computed tomographic angiography, it is necessary to select a perforator based on its diameter. Thus, we emphasize the decision-making process for perforator selection. ICG uptake was measured 5 minutes after anastomosis. De-epithelization was performed after ICG angiography was completed. In bilateral reconstruction, the perforator of the lateral row was selected as often as possible, and the medial row was selected when the actual perforator was unsuitable. Two drains were placed for each breast in the upper pole and the lower pole. Two drains were also placed at the donor site. Intermittent pneumatic compression and low molecular weight heparin were used to prevent deep vein thrombosis. We administered prostaglandin $\mathrm{E}$ for 5 postoperative days to induce vessel dilation.

\section{Assessment of complications}

Postoperative complications were divided into two categories based on the time of occurrence. Complications that occurred 
within 6 months postoperatively were considered early complications, and those occurring after 6 postoperative months were considered late complications. In addition, complications that led to additional revisional surgery were considered major complications, and those handled with conservative treatment only were considered minor complications. Several complications in the implant group were noted, including mastectomy flap necrosis, surgical site infection, hematoma, implant removal, capsular contracture, and cosmetic revisions. Explantation was defined as implant removal due to postoperative complications. Cases of capsular contracture that showed breast animation deformity and pain and muscle contracture around the implant were rated using the Baker scoring system. In this study, scores of 3 or 4 points indicated contracture. Complications among the DIEP patients included mastectomy skin flap necrosis, fat necrosis, nipple necrosis, revisional operation, wound dehiscence, and flap failure.

\section{Assessment of nipple-areolar complex symmetry}

All photographs were taken by a female professional photographer who, at the time of this study, had taken close to 5,000 total clinical pictures of breast cancer patients at our center. Standard photographs show patients from the neck to below the umbilicus while they are undressed above the waist and wear no visible accessories. There are three basic variations of arm positions. In total, 18 (75\%) and 17 (60.7\%) patients who underwent nipple-sparing mastectomy received DIEP and implant reconstruction, respectively. For patients who underwent skin-sparing mastectomy, the center of the skin paddle or the center of the incision line was used to determine the position of the nipple and evaluated. Photographs were taken approximately 1 year later to evaluate symmetry. Illustrations of how the measurements were conducted are shown in Fig. 2. Evaluation of symmetry was performed using three measured points. The distance between the sternal notch (SN) and the right nipple (N1) was indicated as SN-N1, and the distance between the SN and the left nipple (N2) was indicated as SN-N2. The distance between N1 and N2 was marked as N1-N2. The ratio between SN-N1, SN-N2, and N1-N2 was calculated, and a ratio of 1:1:1 was considered to indicate symmetrical breasts [19]. Another evaluation method involved measuring the angle between the horizontal line and the line spanning from N1 to N2. The angle was measured as zero degrees when the nipple-areolar complexes were located in the same position horizontally. Adobe Photoshop CS3 (Adobe Systems Inc., Mountain View, CA, USA) was used to make all of the above measurements.

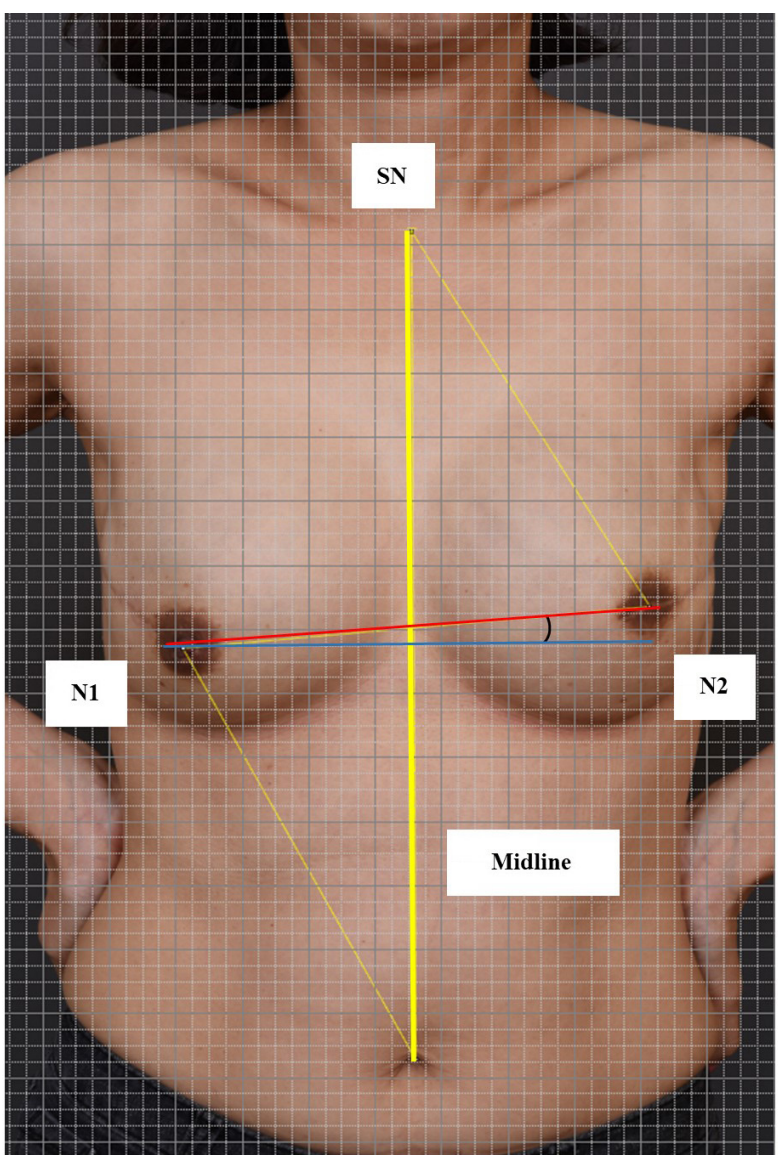

Fig. 2. Depiction of the method for assessing breast symmetry. SN, sternal notch; N1, right nipple; N2, left nipple.

\section{Statistical analysis}

Categorical variables in the groups that underwent reconstruction with implants and DIEP flaps were compared using the chisquare test or Fisher exact test. Continuous variables in the two groups were compared using the t-test or Wilcoxon rank-sum test. For data with a non-normal distribution, the Mann-Whitney test was used. All reported P-values were two-sided, and $\mathrm{P}<0.05$ was considered to indicate statistical significance. All statistical analyses were performed using SPSS version 20.0 (IBM Corp., Armonk, NY, USA).

\section{RESULTS}

Between 2010 and 2020, 52 patients with 104 breast reconstructions were enrolled in our study. Among these, 24 women received bilateral breast reconstruction using a DIEP flap and 26 women received bilateral breast reconstruction using implants (Fig. 3). Patients' demographic characteristics are summarized in Table 1. The average age of the patients was $44.88 \pm 6.7$ and $48.38 \pm 8.28$ years in the DIEP and implant groups, respectively. In the DIEP group, the average BMI was $25.65 \pm 4.10 \mathrm{~kg} / \mathrm{m}^{2}$, 

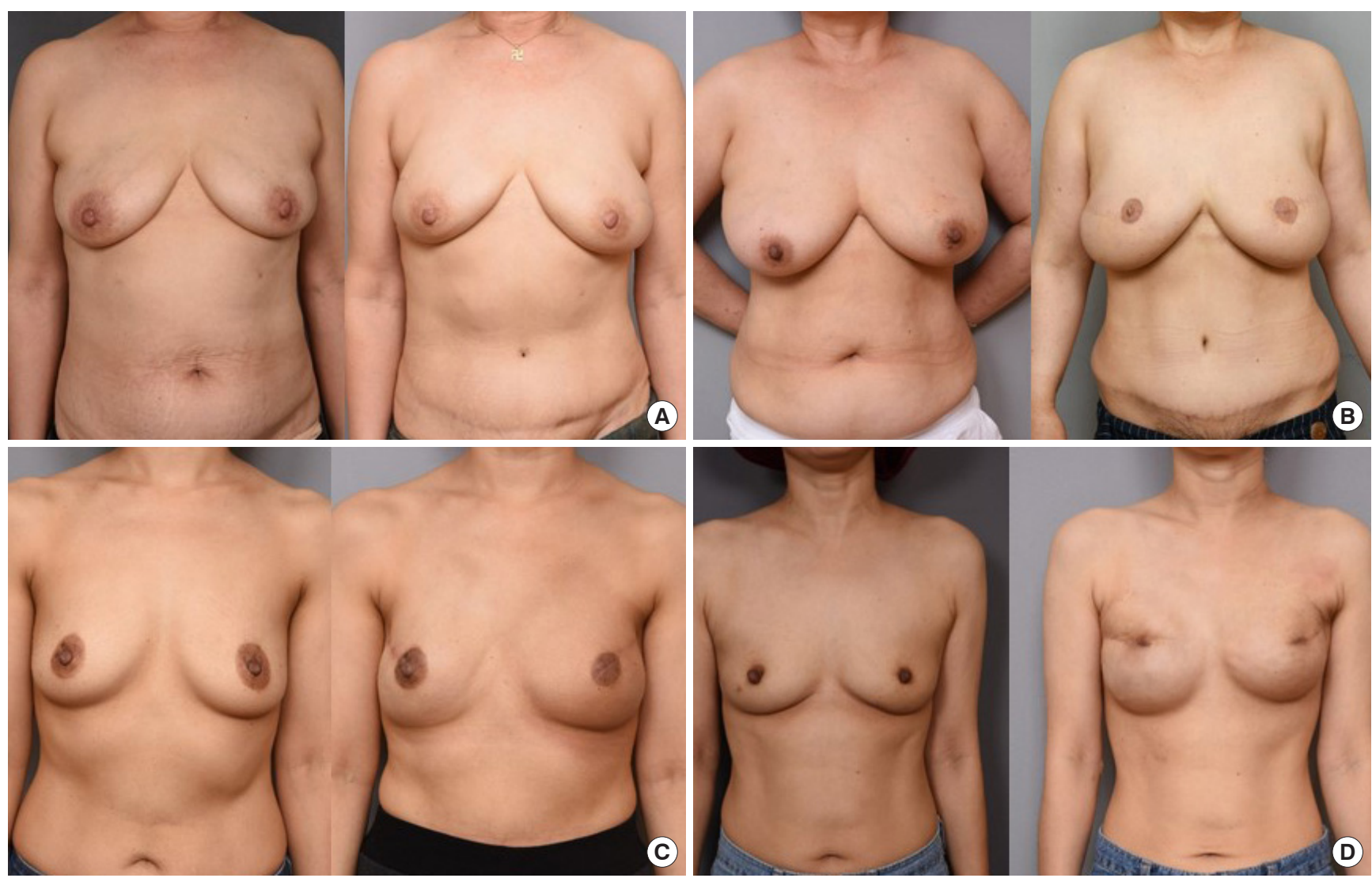

Fig. 3. Representative cases of patients who received bilateral reconstruction. (A) Deep inferior epigastric perforator (DIEP) flap reconstruction case: a 58-year-old patient, preoperative and 8-month postoperative photographs. (B) DIEP flap reconstruction case: a 54-year-old patient, preoperative and 12-month postoperative photographs. (C) Implant reconstruction case: a 63-year-old patient, preoperative and 6-month postoperative photographs. (D) Implant reconstruction case: a 44-year-old patient, preoperative and 12-month postoperative photographs.

Table 1. Demographics of patients in the DIEP and implant groups

\begin{tabular}{lccc}
\hline Characteristic & $\begin{array}{c}\text { DlEP group } \\
(\mathrm{n}=24)\end{array}$ & $\begin{array}{c}\text { Implant group } \\
(\mathrm{n}=26)\end{array}$ & P-value \\
\hline Age $(\mathrm{yr})$ & $44.88 \pm 6.70$ & $48.38 \pm 8.28$ & 0.221 \\
BMl $\left(\mathrm{kg} / \mathrm{m}^{2}\right)$ & $25.65 \pm 4.10$ & $22.82 \pm 4.79$ & 0.011 \\
Height $(\mathrm{m})$ & $1.60 \pm 0.07$ & $1.59 \pm 0.04$ & 0.968 \\
Weight $(\mathrm{kg})$ & $65.18 \pm 11.58$ & $57.87 \pm 12.85$ & 0.022 \\
Hypertension & $1(4.17)$ & $5(19.23)$ & 0.157 \\
Diabetes mellitus & 0 & $1(3.84)$ & 1.000 \\
Current or past smoker & 0 & $1(3.84)$ & 1.000 \\
Premenopausal & $21(87.50)$ & $18(69.23)$ & 0.054 \\
Neoadjuvant chemotherapy & $8(33.33)$ & $7(26.92)$ & 0.508 \\
Adjuvant chemotherapy & $6(25.00)$ & $2(7.69)$ & 0.123 \\
PMRT & $8(33.33)$ & $4(15.38)$ & 0.104 \\
Hormone receptor & $21(87.50)$ & $22(54.61)$ & 1.000 \\
HER2 receptor & $10(41.67)$ & $7(26.92)$ & 0.202 \\
Follow-up period (mo) & $12.93 \pm 6.75$ & $12.39 \pm 9.32$ & 0.599 \\
\hline
\end{tabular}

Values are presented as mean $\pm \mathrm{SD}$ or number $(\%)$.

DIEP, deep inferior epigastric perforator; BMI, body mass index; PMRT, postmastectomy radiation therapy; HER2, human epidermal growth factor receptor 2.

whereas it was $22.82 \pm 4.79 \mathrm{~kg} / \mathrm{m}^{2}$ in the implant group. The average weight of patients in the DIEP group was significantly higher than that of the implant group $(\mathrm{P}=0.011)$. Age, hypertension, diabetes mellitus, smoking history, chemotherapy, premenopausal status, and radiation therapy were similarly distributed among the two groups. The average follow-up period until when photographs were taken was $12.93 \pm 6.75$ months for the DIEP group and $12.39 \pm 9.32$ months for the implant group.

Surgical characteristics are summarized according to the method in Table 2. The average mastectomy specimen weight was $429.36 \pm 211.51 \mathrm{~g}$ and $327.19 \pm 214.36 \mathrm{~g}$ in the DIEP and implant groups, respectively. Among patients who underwent DIEP flap reconstruction, the average weight of the mastectomy flap was significantly higher than that of patients who received implants $(\mathrm{P}=0.049)$. The average flap weight of the reconstructed breasts in the DIEP group was $417.43 \pm 152.50 \mathrm{~g}$, which was significantly higher than that of the implant group (311.07 \pm $108.72 \mathrm{~g} ; \mathrm{P}=0.010$ ). The ratio of the mean reconstruction flap (or implant) weight to the mastectomy specimen weight was $1.17 \pm 0.70$ in the DIEP group and $1.12 \pm 0.39$ in the implant group, indicating that sufficient reconstruction was achieved. All implants used were textured. There was a significant difference 
Table 2. Operative characteristics of the DIEP and implant groups

\begin{tabular}{|c|c|c|c|}
\hline Characteristics & $\begin{array}{l}\text { DIEP group } \\
(n=24)\end{array}$ & $\begin{array}{l}\text { Implant group } \\
(n=26)\end{array}$ & P-value \\
\hline Treatment for affected breast & & & 0.143 \\
\hline Bilateral & $18(75.00)$ & $24(92.3)$ & \\
\hline Unilateral & $6(25.00)$ & $2(7.14)$ & \\
\hline Mastectomy type & & & 0.551 \\
\hline Both NSM & $18(75.00)$ & $15(57.69)$ & \\
\hline $\mathrm{NSM}+\mathrm{SSM}$ & $4(16.67)$ & $6(21.43)$ & \\
\hline Both SSM & $2(8.33)$ & $5(17.86)$ & \\
\hline Axillary dissection & $5(20.83)$ & $3(11.53)$ & 0.576 \\
\hline \multicolumn{4}{|l|}{ Mastectomy weight (g) } \\
\hline Left & $434.41 \pm 215.79$ & $326.50 \pm 215.04$ & 0.038 \\
\hline Right & $424.31 \pm 213.30$ & $328.15 \pm 215.95$ & 0.063 \\
\hline Mean & $429.36 \pm 211.51$ & $327.19 \pm 214.36$ & 0.049 \\
\hline \multicolumn{4}{|l|}{$\begin{array}{l}\text { Reconstruction flap (g) or } \\
\text { implant (cc) weight }\end{array}$} \\
\hline Left & $420.66 \pm 150.00$ & $311.32 \pm 109.10$ & 0.009 \\
\hline Right & $414.21 \pm 156.41$ & $310.38 \pm 108.48$ & 0.029 \\
\hline Mean & $417.43 \pm 152.50$ & $311.15 \pm 108.72$ & 0.010 \\
\hline $\begin{array}{l}\text { Ratio of reconstruction flap or } \\
\text { implant weight to } \\
\text { mastectomy weight }\end{array}$ & $1.17 \pm 0.70$ & $1.12 \pm 0.39$ & 0.741 \\
\hline Operation time (min) & $454.67 \pm 130.78$ & $127.92 \pm 35.73$ & $<0.001$ \\
\hline POD (day) & $6.67 \pm 1.13$ & $5.23 \pm 1.31$ & $<0.001$ \\
\hline
\end{tabular}

Values are presented as the number (\%) or mean \pm SD.

DIEP, deep inferior epigastric perforator; NSM, nipple-sparing mastectomy; SSM, skin-sparing mastectomy; POD, postoperative day.

$(\mathrm{P}<0.001)$ in the average operation time between the two groups (454.67 \pm 130.78 minutes for the DIEP group and $127.92 \pm$ 35.73 minutes for the implant group). The average hospitalization period of DIEP patients was $6.67 \pm 1.13$ days, which was significantly longer $(\mathrm{P}<0.001)$ than that of patients who received implants (5.23 \pm 1.31 days).

\section{Complications}

In the implant group, hematoma, seroma, surgical site infection, implant shift and rotation, nipple necrosis, mastectomy flap necrosis, and capsular contracture were recorded. Similarly, in the DIEP group, fat necrosis, fluid collection, nipple necrosis, dehiscence of the wound, surgical site infection, and revision surgery for blood vessels were recorded.

In the implant group, 19 patients (36.53\%) experienced early complications, which was significantly higher than the rate of early complications in the DIEP group $(\mathrm{P}=0.049)$. Although it was higher in the implant group, the rate of late complications was not significantly different between the two groups (17.3\% in the implant group and $4.16 \%$ in the DIEP group, $\mathrm{P}=0.057)$.

Minor complications were observed in 21 cases $(40.38 \%)$ in the implant group and only four cases $(8.32 \%)$ in the DIEP group $(\mathrm{P}<0.001)$. The rate of major complications was similar
Table 3. Major and minor complications in the DIEP and implant groups

\begin{tabular}{lccc}
\hline \multirow{2}{*}{ Complications } & \multicolumn{2}{c}{ Group, No. $(\%)$} & \\
\cline { 2 - 3 } & DIEP $(\mathrm{n}=48)$ & Implant $(\mathrm{n}=52)$ & \\
\hline Minor complications & $4(8.32)$ & $21(40.38)$ & 0.003 \\
Major complications & $8(16.66)$ & $7(13.46)$ & 0.579 \\
Late complications & $2(4.16)$ & $9(17.3)$ & 0.057 \\
Early complications & $8(16.66)$ & $19(36.53)$ & 0.049 \\
Recurrence & 0 & $3(5.76)$ & 0.240 \\
\hline
\end{tabular}

DIEP, deep inferior epigastric perforator.

between the DIEP and implant groups, with eight cases $(16.66 \%)$ and seven cases $(13.46 \%)$, respectively $(P=0.579)$. The differences in the recurrence rates of the two groups were not significant $(\mathrm{P}=0.240)$. Given the overall rate of complications, the proportion of major complications in the DIEP group was high. Nipple necrosis and fat necrosis accounted for a large proportion of complications ( $34 \%$ and $25 \%$, respectively). In the implant group, the proportion of minor complications was higher than that of major complications, with seroma being the most common complication (27\%) followed by implant shift or rotation (23\%) (Table 3, Fig. 4).

\section{Symmetry assessment}

The average ratio of SN1-N1/SN2-N2 (or SN2-N2/SN1-N1 if SN1-N1 was shorter than SN2-N2) was $0.96 \pm 0.02$ in the DIEP group and $0.97 \pm 0.03$ in the implant group. The average ratio of the distance between SN-N to N1-N2 was $0.94 \pm 0.07$ in the DIEP group and $1.04 \pm 0.10$ in the implant group, with the difference being statistically significant $(\mathrm{P}=0.002)$. The average angle between N1-N2 and the horizontal line was $1.09^{\circ} \pm 0.71^{\circ}$ in the DIEP group and $1.75^{\circ} \pm 1.45^{\circ}$ in the implant group, which also reflected a statistically significant difference $(\mathrm{P}=0.046)$ (Table 4, Fig. 2).

\section{DISCUSSION}

The number of patients with unilateral breast cancer who are at risk of developing cancer in the opposite breast is increasing $[11,20]$. A risk-reducing mastectomy is when the contralateral normal breast of a patient diagnosed with unilateral breast cancer is resected $[13,16,17]$. An increasingly large proportion of women with unilateral breast cancer are undergoing bilateral mastectomy due to anxiety about the possibility of developing cancer in the other breast [3]. In particular, among patients who carry the $B R C A$ mutation, the incidence of breast cancer is high, and there is substantial demand for prophylactic treatment [13]. In 2013, Angelina Jolie, one of the most famous actresses in Hollywood, disclosed to the New York Times that she had undergone 

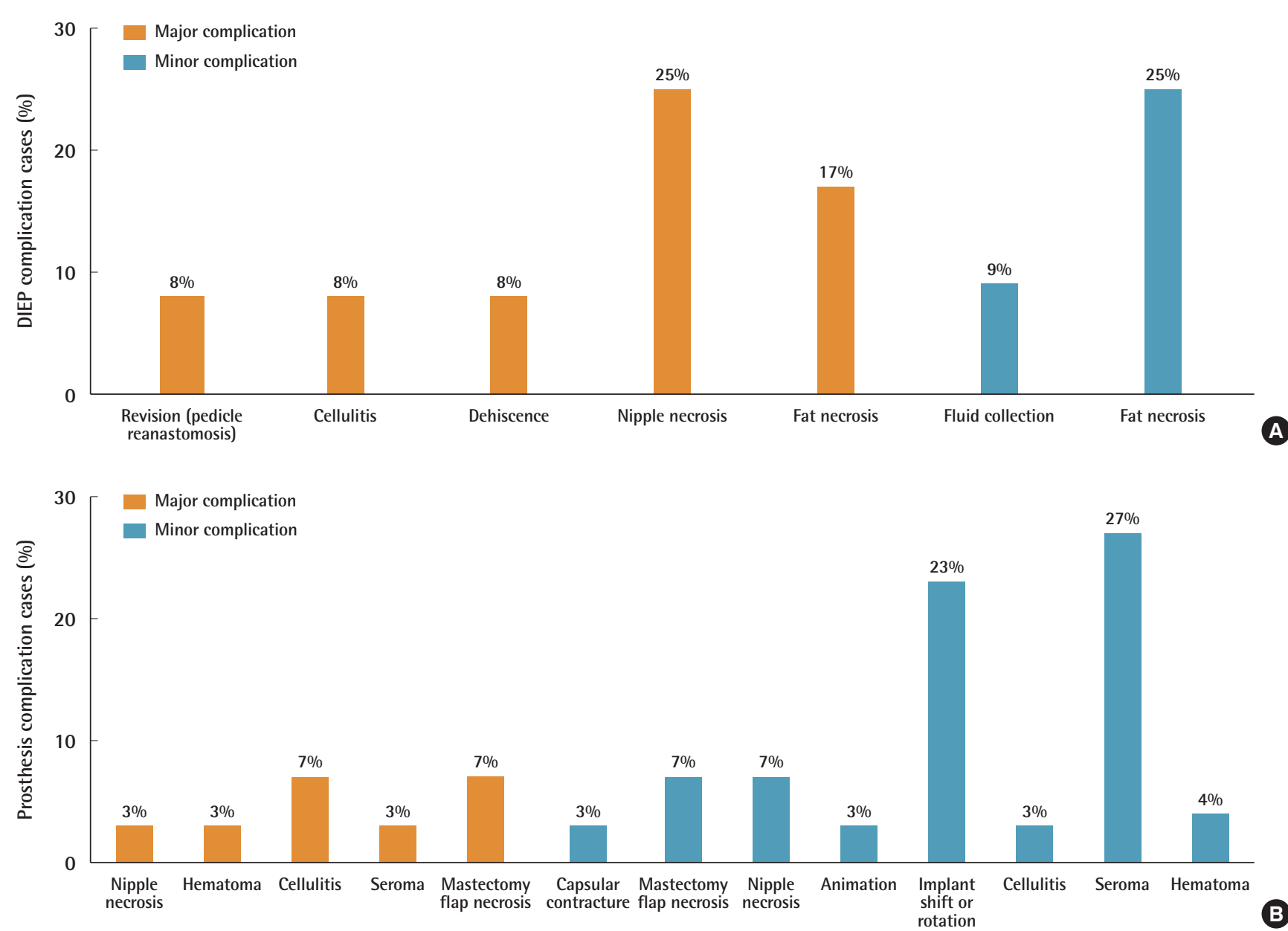

Fig. 4. Major and minor complications in the deep inferior epigastric perforator (DIEP) and implant groups (A, B).

Table 4. Breast symmetry measurements in the DIEP and implant groups

\begin{tabular}{lccc}
\hline \multirow{2}{*}{ Variable } & \multicolumn{2}{c}{ Group, average \pm SD } & \\
\cline { 2 - 3 } & $\begin{array}{c}\text { DIEP } \\
(\mathrm{n}=24)\end{array}$ & $\begin{array}{c}\text { Implant } \\
(\mathrm{n}=26)\end{array}$ & P-value \\
\hline Ratio of shorter SN-N to longer SN-N & $0.96 \pm 0.02$ & $0.97 \pm 0.03$ & 0.177 \\
Ratio of N1-N2 to longer SN-N & $0.94 \pm 0.07$ & $1.04 \pm 0.10$ & 0.002 \\
Angle between the N1-N2 line and & $1.09 \pm 0.71$ & $1.75 \pm 1.45$ & 0.046 \\
\hline
\end{tabular}

DIEP, deep inferior epigastric perforator; SN, sternal notch; N, nipple; N1, left nipple; N2, right nipple.

a risk-reducing bilateral mastectomy after finding out that she was a BRCA1 mutation carrier [21]. This decision by a highprofile celebrity prompted the general public to acquire more information about the BRCA gene and encouraged people to undergo genetic testing and make a decision regarding risk-reducing mastectomy. Interestingly, this phenomenon has been termed the "Jolie effect." Owing to these reasons, there is an increasing worldwide trend in which more women are receiving therapeutic mastectomy, and an increase in the rate of contralat- eral prophylactic mastectomy has been observed [20,21].

Therefore, analyzing and evaluating the outcomes of bilateral reconstruction at this time will provide further evidentiary support regarding this growing trend. At the time of this study, however, only a few studies have analyzed bilateral reconstruction $[9,22,23]$. No direct comparisons have been made between autologous and implant reconstruction. It is widely agreed that reconstruction using implants is easy and efficient. Additionally, while DIEP flap reconstruction allows women to maintain a natural-looking breast, the operation time is long. There is no data directly comparing the outcomes of these two procedures, however. Hence, this study aimed to fill this research gap.

Based on our demographic data, the average BMI was higher in the DIEP group than in the implant group. A possible reason for this might have been that obese patients were encouraged to choose autologous reconstruction.

Both approaches can be considered acceptable and effective surgical methods since the rate of major complications did not differ significantly between the two groups. However, minor complications were more common than major complications in 
the implant group, and they were significantly more common in the implant group than in the DIEP group. Most implant complications involved malpositioning, which signifies the difficulty of keeping both implants in their original positions. Therefore, the implant group had poor outcomes in terms of symmetry. However, since all of the implants in our study were placed in the subpectoral plane, muscle action may have had a disproportionate effect on the position of implants. Given the increasing tendency to place implants in the prepectoral plane $[24,25]$, outcomes may differ for prepectoral reconstruction, which is a possibility that should be explored in future studies. In addition, due to the frequency of seroma, many patients needed to undergo seroma aspiration, which is an undesirable outcome of implant reconstruction. In the DIEP group, however, inefficiency poses an issue due to the length of the operation itself, which was almost four times longer than implant surgery. The possibility of flap revision and flap failure must also be considered. DIEP reconstruction yielded better cosmetic outcomes than implant reconstruction (Fig. 3) since reconstruction on both sides of the breast allowed symmetry in the nipple-areolar complex to be maintained, resulting in a natural-looking breast.

The symmetry indicator can roughly estimate the direction in which the nipple-areolar complex moves progressively between the implant and the DIEP. In implants with an N1-N2/SN-N1 ratio of greater than 1, the nipple-areolar complex tends to move laterally, while DIEP flaps with an N1-N2/SN-N1 ratio of less than 1 tend to correspond to a small change in the nipple-areolar complex toward the medial side. This is not an indicator that can objectively quantify symmetry. The number of patients who underwent nipple-sparing mastectomy was 18 (75\%) and $17(60.7 \%)$ in the DIEP and implant groups, respectively. In skin-sparing mastectomy, the center of the skin paddle or the center of the incision line was used to indicate the nipple position and evaluated accordingly, which may have led to errors when determining the exact nipple position.

Based on our results, bilateral autologous reconstruction can achieve good aesthetic results without leading to many minor complications. Hence, patients who receive this procedure will spend less time visiting outpatient clinics after surgery. Bilateral implant reconstruction has the advantage of being very efficient, but it may result in frequent outpatient visits and require treatment for minor complications after the initial surgery. Surgeons should provide this information to patients to help them choose their preferred surgical reconstruction method. In the near future, the lengthy operation time of DIEP flap reconstruction, which is its biggest drawback, will be shortened dramatically due to the emergence of coupler devices [26], the development of micro-instruments [26], and advances in perioperative imag- ing modalities $[27,28]$. In addition, the preferred plane for the placement of implants is gradually changing to the prepectoral plane [24], which helps to avert breast deformations following post-mastectomy radiation therapy - a highly undesirable outcome of implant reconstruction $[10,14,29]$. In addition, the quality of $\mathrm{ADM}$ is also improving. Thus, the shortcomings of implants are being actively addressed. Therefore, no surgical methods can be considered absolutely unfavorable or advantageous with regard to bilateral breast reconstruction.

ICG angiography is a necessary procedure even for slim patients. The BMI values of Korean patients have changed significantly compared to those values in the past. The number of breast cancer patients with a high BMI $\left(30-35 \mathrm{~kg} / \mathrm{m}^{2}\right)$ is increasing. Additionally, the perforator is often weak and tiny in slim patients, and tissue in the abdomen tends to be thin. However, since the volume of the breast is relative to the abdomen, the abdomen marking is often widened. Therefore, for Asian patients, ICG is reasonable, and this short and simple procedure is routinely conducted at our center.

There were some limitations to this study. First, data were collected on Asian patients who tend to have a relatively slim body habitus. Since obesity is a key factor related to complications from breast reconstruction $[9,10,15,29,30]$, the associated complications found in this study may be slightly different for a population with a higher prevalence of obesity. Second, the sample size was small, partly due to the treatment policy of our center to attempt breast-conserving surgery to the extent possible. Third, it was difficult to equalize factors for comparison, especially for complications between the DIEP and implant groups, due to differences in the assessment factors evaluated during the follow-up period. In addition, a qualified subjective assessment such as the BREAST-Q was not conducted in addition to an objective assessment. Lastly, in skin-sparing mastectomy, the center of the skin paddle or the center of the incision line was considered to indicate the location of the nipple and then evaluated. As a result, errors in determining the exact nipple position may have occurred.

In conclusion, both DIEP flap and implant reconstructions are viable surgical methods with their own advantages and disadvantages. When using implants for bilateral reconstruction, implant positioning, asymmetry features, and complications such as seroma should be considered and monitored. Although the surgical burden is small for implant reconstruction, frequent and intensive follow-up is often required after the initial surgery. DIEP flap reconstruction involves a long operation time and carries the risk of flap failure. However, DIEP flap reconstruction provides positive cosmetic results, and follow-up treatments are typically not required. Nevertheless, patients should 
be informed, and a reconstruction method should be recommended that is most suitable for them.

\section{NOTES}

\section{Conflict of interest}

No potential conflict of interest relevant to this article was reported.

\section{Ethical approval}

The study was approved by the Institutional Review Board of Asan Medical Center (IRB No. S2020-2202-0001) and performed in accordance with the principles of the Declaration of Helsinki. Written informed consent was obtained.

\section{Patient consent}

The patients provided written informed consent for the publication and the use of their images.

\section{Author contribution}

Conceptualization: HH Han, JS Eom. Data curation: JS Eom. Formal analysis: HH Han, JM Choi. Methodology: HH Han. Writing - original draft: HH Han, JM Choi. Writing - review \& editing: HH Han.

\section{ORCID}

Hyun Ho Han

Jin Mi Choi

Jin Sup Eom https://orcid.org/0000-0001-7072-9882 https://orcid.org/0000-0002-1855-7910 https://orcid.org/0000-0003-3229-2012

\section{REFERENCES}

1. Carbine NE, Lostumbo L, Wallace J, et al. Risk-reducing mastectomy for the prevention of primary breast cancer. Cochrane Database Syst Rev 2018;4:CD002748.

2. Schneider AP 2nd, Zainer CM, Kubat CK, et al. The breast cancer epidemic: 10 facts. Linacre Q 2014;81:244-77.

3. DeSantis CE, Ma J, Gaudet MM, et al. Breast cancer statistics, 2019. CA Cancer J Clin 2019;69:438-51.

4. Nealon KP, Sobti N, Gadd M, et al. Assessing the additional surgical risk of contralateral prophylactic mastectomy and immediate breast implant reconstruction. Breast Cancer Res Treat 2020;179:255-65.

5. Narod SA. Bilateral breast cancers. Nat Rev Clin Oncol 2014; 11:157-66.

6. Sakai T, Ozkurt E, DeSantis S, et al. National trends of synchronous bilateral breast cancer incidence in the United States. Breast Cancer Res Treat 2019;178:161-7.
7. Taylor EM, Wilkins EG, Pusic AL, et al. Impact of unilateral versus bilateral breast reconstruction on procedure choices and outcomes. Plast Reconstr Surg 2019;143:1159e-1168e.

8. Yueh JH, Slavin SA, Adesiyun T, et al. Patient satisfaction in postmastectomy breast reconstruction: a comparative evaluation of DIEP, TRAM, latissimus flap, and implant techniques. Plast Reconstr Surg 2010;125:1585-95.

9. Drazan L, Vesely J, Hyza P, et al. Bilateral breast reconstruction with DIEP flaps: 4 years' experience. J Plast Reconstr Aesthet Surg 2008;61:1309-15.

10. Hu ES, Pusic AL, Waljee JF, et al. Patient-reported aesthetic satisfaction with breast reconstruction during the long-term survivorship Period. Plast Reconstr Surg 2009;124:1-8.

11. Yun J, Jeong HH, Cho J, et al. Weight analysis of mastectomy specimens and abdominal flaps used for breast reconstruction in Koreans. Arch Plast Surg 2018;45:246-52.

12. King TA, Sakr R, Patil S, et al. Clinical management factors contribute to the decision for contralateral prophylactic mastectomy. J Clin Oncol 2011;29:2158-64.

13. Breast Cancer Linkage Consortium. Cancer risks in BRCA2 mutation carriers. J Natl Cancer Inst 1999;91:1310-6.

14. Isaksson K, Arver B, Bottai M, et al. Bilateral risk-reducing mastectomies with implant-based reconstructions followed long term: a consecutive series of 185 patients. World J Surg 2019;43:2262-70.

15. Rosson GD, Magarakis M, Shridharani SM, et al. A review of the surgical management of breast cancer: plastic reconstructive techniques and timing implications. Ann Surg Oncol 2010;17:1890-900.

16. Thorat MA, Balasubramanian R. Breast cancer prevention in high-risk women. Best Pract Res Clin Obstet Gynaecol 2020;65:18-31.

17. Tuttle TM, Habermann EB, Grund EH, et al. Increasing use of contralateral prophylactic mastectomy for breast cancer patients: a trend toward more aggressive surgical treatment. J Clin Oncol 2007;25:5203-9.

18. Ozturk A, Alco G, Sarsenov D, et al. Synchronous and metachronous bilateral breast cancer: a long-term experience. J BUON 2018;23:1591-600.

19. Penn J. Breast reduction. Br J Plast Surg 1955;7:357-71.

20. Clark K. Efficacy of bilateral prophylactic mastectomy in women with a family history of breast cancer. J Insur Med 1999;31:41-3.

21. Liede A, Cai M, Crouter TF, et al. Risk-reducing mastectomy rates in the US: a closer examination of the Angelina Jolie effect. Breast Cancer Res Treat 2018;171:435-42.

22. Holoyda KA, Simpson AM, Ye X, et al. Immediate bilateral breast reconstruction using abdominally based flaps: an 
analysis of the nationwide inpatient sample database. J Reconstr Microsurg 2019;35:594-601.

23. Guerra AB, Metzinger SE, Bidros RS, et al. Bilateral breast reconstruction with the deep inferior epigastric perforator (DIEP) flap: an experience with 280 flaps. Ann Plast Surg 2004;52:246-52.

24. Elswick SM, Harless CA, Bishop SN, et al. Prepectoral implant-based breast reconstruction with postmastectomy radiation therapy. Plast Reconstr Surg 2018;142:1-12.

25. Graziani C, Panico C, Botti G, et al. Subperiosteal midface lift: its role in static lower eyelid reconstruction after chronic facial nerve palsy. Orbit 2011;30:140-4.

26. Ardehali B, Morritt AN, Jain A. Systematic review: anastomotic microvascular device. J Plast Reconstr Aesthet Surg 2014;67:752-5.

27. Anker AM, Prantl L, Strauss C, et al. Assessment of DIEP flap perfusion with intraoperative indocyanine green fluorescence imaging in vasopressor-dominated hemodynamic support versus liberal fluid administration: a randomized controlled trial with breast cancer patients. Ann Surg Oncol 2020;27:399-406.

28. Vargas CR, Koolen PG, Ho OA, et al. Preoperative CT-angiography in autologous breast reconstruction. Microsurgery 2016;36:623-7.

29. Lemaine V, Schilz SR, Van Houten HK, et al. Autologous breast reconstruction versus implant-based reconstruction: how do long-term costs and health care use compare? Plast Reconstr Surg 2020;145:303-11.

30. van Veen MM, Dijkstra PU, Werker P. A higher quality of life with cross-face-nerve-grafting as an adjunct to a hypoglossal-facial nerve jump graft in facial palsy treatment. J Plast Reconstr Aesthet Surg 2017;70:1666-74. 